\title{
Hematological and Inflammatory Parameters to Predict the Prognosis in COVID-19
}

\author{
Hakan Keski ${ }^{1}$
}

Received: 15 August 2020/Accepted: 9 February 2021/Published online: 27 February 2021

(C) Indian Society of Hematology and Blood Transfusion 2021

\begin{abstract}
We aimed to evaluate the predictive ability of hematological and inflammatory parameters for mortality in COVID-19 patients. This was a retrospective study of hospitalized COVID-19 patients over 18 years old between March 2020 and May 2020. Patients were diagnosed to have COVID-19 based either on chest computed tomography findings or reverse transcriptase-polymerase chain reaction test. Age, gender, chronic medical conditions, and laboratory values including hemogram parameters (white blood cell, neutrophil, lymphocyte, and platelet counts), neutrophil to lymphocyte ratio, D-dimer, ferritin, fibrinogen, C-reactive protein, procalcitonin, prothrombin time, activated partial thromboplastin time and the international normalized ratio were recorded. Overall, we included 302 patients. Of these, 148 patients were male; the male to female ratio was 0.961 . The mean age of the entire study cohort was $57.1 \pm 17.6$ years. The most common chronic medical condition was hypertension (38.1\%). Half of the patients received low molecular weight heparin. During the study period, 25 patients $(8.2 \%)$ died. White blood cell count and neutrophil count were significantly higher, whereas lymphocyte count was significantly lower in the deceased patients. The median neutrophil to lymphocyte ratio was 11.6 in the deceased patients, it was significantly higher than the surviving patients $(p<0.001)$. The values of C-reactive protein, procalcitonin, D-dimer, and ferritin were significantly higher among the deceased patients. Prothrombin time, activated partial thromboplastin time
\end{abstract}

Hakan Keski

keski1976@gmail.com

1 Department of Hematology, Umraniye Training and Research Hospital, Elmalıkent Mh. Adem Yavuz Cd. No: 1 Ümraniye, İstanbul, Turkey and the international normalized ratio were significantly longer in the deceased group compared with the surviving group. Logistic regression analysis showed age $>65$ years, neutrophil to lymphocyte ratio, activated partial thromboplastin time, and hypertension as the independent predictors of mortality. The rate of abnormal inflammatory and hematologic-coagulation parameters increased with the COVID-19 severity. Age $>65$ years, hypertension, activated partial thromboplastin time and neutrophil to lymphocyte ratio were the independent predictors of mortality.

Keywords Coagulation - COVID-19 - Inflammation · Mortality $\cdot$ Neutrophil-to-lymphocyte ratio

\section{Introduction}

Severe acute respiratory syndrome coronavirus 2 (SARSCoV-2) infection, named coronavirus disease 2019 (COVID-19), created a pandemic [1]. One of the striking features of COVID-19 was its systemic nature, although the virus's primary target was the respiratory tract. Many patients, particularly the elderly and having comorbid conditions, succumbed to multiorgan failure manifested with laboratory and clinical dysfunction in several organ systems, including heart, kidneys, coagulation system, and others [2-4].

It is of crucial importance to be able to predict which patients would develop a severe disease and which patients would have a higher risk of death to allocate limited resources better. To this end, several hematological and biochemical (particularly inflammatory) parameters, as well as presenting symptoms, have been examined to understand their ability to predict the severity of disease and mortality outcomes [2, 5-7]. 
Hematological parameters, including peripheral blood counts and coagulation tests, have become prominent in predicting the severity of the COVID-19 from the outset. Moreover, disseminated intravascular coagulation (DIC) was common among non-survived COVID-19 patients [8]. Thus, hematological parameters are of significant importance for treatment decisions in severe COVID-19 patients.

Several hematological parameters have been reported to be disturbed in patients with COVID-19, starting from the disease's early phases [9-12]. Besides, therapeutic anticoagulation in high-risk patients proved to be effective in reducing mortality [13]. Inflammation and coagulation cascades have a cross-talk in COVID-19 patients [14].

Thus, we aimed to evaluate the prevalence of abnormal hematological parameters (including coagulation tests and peripheral blood counts) along with inflammatory markers and their ability to predict mortality in patients with COVID-19.

\section{Materials and Methods}

This study was a retrospective analysis of the COVID-19 patients hospitalized in COVID-19 inpatient wards at Umraniye Education and Research Hospital between March 2020 and May 2020. Patients were diagnosed to have COVID-19 based on either the reverse-transcription quantitative polymerase chain reaction (RT-qPCR) tests applied on respiratory secretions obtained via nasal swabs or the chest computed tomography (CT) findings. The presence of single or multiple diffuse ground-glass appearance associated with consolidation was regarded as the positive CT findings for COVID-19 infection [15-17]. The positive and negative RT-qPCR results were detected in $270(89.4 \%)$ and 32 patients (10.6\%). The diagnosis was confirmed via positive CT findings seen in 225 patients (74.8\%). Patients over 18 years old were included in the study. Patients with a diagnosis of COVID-19 infection were excluded if they were followed up as an outpatient.

Age, gender, chronic medical conditions, and laboratory results, including hemogram parameters [white blood cell (WBC) count, neutrophil count, lymphocyte count, thrombocyte count, hemoglobin, red cell distribution width (RDW), and mean platelet volume (MPV)], D-dimer, lactate dehydrogenase (LDH), ferritin, fibrinogen, C-reactive protein $(\mathrm{CRP})$, procalcitonin, prothrombin time (PT), the international normalized ratio (INR), and activated partial thromboplastin time (aPTT) were retrieved from the hospital electronic database system.

This study was performed in line with the principles of the Declaration of Helsinki. The local ethics committee approved the study. Informed consent was obtained from all participants included in the study.

\section{Statistical Analysis}

Descriptive data derived from the study were presented as mean \pm standard deviation or median and interquartile ranges of 25th and 75th percentiles (IQR 1-3) or the minimum and maximum depending on the distribution of continuous variables. Categorical variables were given as number and percentage. The normal distribution of numerical variables was checked with the KolmogorovSmirnov and the Shapiro-Wilks tests. In comparison of the two independent groups, the independent samples $t$-test was used when the numerical variables had a normal distribution, or the Mann-Whitney U test was used when the numerical variables did not have normal distribution. The differences between the categorical variables were compared with the Pearson chi-square, Fisher's Exact, and Fisher-Freeman-Halton tests. The temporal relationships between the survival rates and white blood cell count, neutrophil count, lymphocyte count, neutrophil-to-lymphocyte ratio, procalcitonin, prothrombin time, aPTT, and INR were evaluated via Kaplan-Meier survival analysis.

The analysis of the receiver operating characteristic (ROC) curve associated with area under curve (AUC) was used to discover the optimal cut-off values of the levels of the laboratory parameters to predict the development of mortality in the study group. AUC was interpreted as excellent if $0.9<$ AUC $<1$, good if $0.8<$ AUC $<0.9$, fair if $0.7<$ AUC $<$ 0.8 , poor if $0.6<$ AUC $<0.7$, and fail if $0.5<$ AUC $<0.6$.

Univariate and multivariate Cox regression analyses were used to evaluate the independent predictors of mortality. The selected variables in the multivariate model were determined based on the significant variables in the univariate analyses. The independent variables that showed multivariate multicollinearity with significant correlation coefficients was not analyzed in the model.

Statistical analyses were performed using the Jamovi project (Version 1.6.3) and JASP (Version 0.13.1). A $p$ value below 0.05 was accepted as statistically significant.

\section{Results}

\section{Clinical and Demographic Features and Laboratory Results of the Entire Study Cohort}

Overall, we included 302 patients who were hospitalized with the diagnosis of COVID-19. Of these, 148 patients were male. The male to female ratio was 0.961 . The mean age of the entire study cohort was $57.1 \pm 17.6$ years. Most of the patients $(85.8 \%)$ were hospitalized in a dedicated COVID-19 hospital ward; the rest showed a need for stay at the intensive care unit (ICU) during or at the outset of their hospitalization. The most common chronic medical 
condition among the patients was hypertension (38.1\%). Clinical and demographic characteristics and laboratory results of the entire study cohort are shown in Table 1 .

\section{Comparison of Clinical and Laboratory Features of the Deceased and Surviving Patients}

During the study period, 25 patients $(8.2 \%)$ died. The deceased patients were significantly older than those who survived (73.4 \pm 13.2 vs. $55.6 \pm 17.2$ years, respectively, $p<0.001$ ). All of these patients except one were hospitalized in the ICU. Ninety-six percent of the deceased patients had at least one chronic medical condition, whereas $63.2 \%$ of the surviving patients had a comorbid disorder $(p=0.002)$. Diabetes mellitus, hypertension, and coronary artery disease were significantly more common among the deceased than in the survivors.

White blood cell count and neutrophil count were significantly higher, whereas lymphocyte count was significantly lower in the deceased patients. Besides, MPV, D-dimer, ferritin, and fibrinogen values were significantly higher, and PT and aPTT were significantly longer in deceased patients. Table 1 summarizes the comparison of the clinical features and laboratory results of the deceased and surviving patients.

We also examined the data as the percentages above the normal reference range for the entire group, the deceased patients, and the surviving patients (Table 2). Overall, the number of patients with leukocytosis and neutrophilia were significantly higher in the deceased group than the surviving group. Interestingly, $13.7 \%$ and $9 \%$ of the patients had leukopenia and neutropenia among the surviving patients, respectively. On the other hand, there was no neutropenia and only one leukopenia in the deceased group. While leukopenia and neutropenia were significantly more common in the surviving patients, lymphopenia was significantly more frequent among the deceased $(p<0.001$, for all). Thrombocytopenia frequency was comparable between both groups.

To determine cut-off values, ROC analysis using the sensitivities and specificities based on the development of mortality revealed that there were the optimal cut-off values for each laboratory parameter except for platelet count with statistical significance. The largest AUC for procalcitonin was 0.894 with the cut-off value of $>0.11$ (sensitivity $=96.0 \%$ and specificity $=77.26 \%$ ). The second highest AUC of 0.861 was observed in NLR with a cut-off value of $>8.59$ (sensitivity $=72.0 \%$ and specificity $=88.45 \%$ ). The predictive power of procalcitonin, NLR, D-Dimer, neutrophil count, ferritin and CRP for mortality were regarded as good. The corresponding sensitivity and specificities of all laboratory parameters under the optimal cut-off values are given in Table 3.

Percentages of patients with results above the normal range for inflammatory markers (C-reactive protein, ferritin, procalcitonin) were significantly higher in the deceased group than the surviving group. All patients $(100 \%)$ had elevated serum procalcitonin levels in the deceased group. Higher than normal serum D-dimer values were observed in $63.9 \%$ and $96 \%$ of the surviving and deceased patients, respectively $(p=0.002)$. Similarly, the percentage of patients with elevated fibrinogen values was higher in the deceased patient group than in the surviving group. Prolonged coagulation tests (increased aPTT and PT) were also more common among the deceased patients.

\section{Survival Analysis}

The Kaplan-Meier survival curves revealed that there were significant associations between mortality and WBC, neutrophil, and lymphocyte counts, NLR, procalcitonin, PT, aPTT, and INR $(p<0.05$ for all) (Fig. 1). Patients with lymphopenia had significantly lower survival rates than patients with normal lymphocyte counts $(p=0.042)$. Patients with higher WBC, neutrophil count, and procalcitonin levels had significantly shorter survival $(p=0.007$, $p<0.001$, and $p<0.001$, respectively). When we grouped all patients based on NLR as those above and below median NLR value (3.07), patients with above-the-median NLR had a significantly higher mortality rate than the others ( $p=0.001)$. Finally, patients with prolonged PT, aPTT, and INR had significantly lower survival rates $(p=0.008$, $p<0.001$ and $p=0.012$, respectively).

\section{Univariate and Multivariate Cox Regression Analysis to Determine Independent Predictors of Mortality}

We included age (as a categorical variable below and above 65 years), presence of hypertension, chronic kidney disease, diabetes mellitus, congestive heart failure, and the indicators such as platelet count, MPV, NLR, CRP, procalcitonin, ferritin, fibrinogen, prothrombin time, and aPTT in the univariate Cox regression analysis to determine the predictors of mortality. In the univariate analysis, being older than 65 years, the presence of hypertension, NLR, CRP, procalcitonin, ferritin, prothrombin time, and aPTT emerged as independent predictors of mortality. Multivariate Cox regression analysis revealed that ages above 65 years, NLR, aPTT, and hypertension remained independent predictors of mortality (Table 4).

\section{Discussion}

Our findings showed the significant differences in demographic and clinical variables, hemostatic biomarkers, coagulation tests, and inflammatory parameters between 
Table 1 Comparison of clinical and demographic characteristics and laboratory results of the deceased and surviving patients

\begin{tabular}{|c|c|c|c|c|}
\hline & \multirow[t]{2}{*}{ Overall $(\mathrm{n}=302)$} & \multicolumn{2}{|l|}{ Survival status } & \multirow[t]{2}{*}{$p$-value } \\
\hline & & Deceased $(n=25)$ & Surviving $(\mathrm{n}=277)$ & \\
\hline Age, years & $57.1 \pm 17.6$ & $73.4 \pm 13.2$ & $55.6 \pm 17.2$ & $<0.001$ \\
\hline \multicolumn{5}{|l|}{ Sex } \\
\hline Male & $148(49.0)$ & $12(48.0)$ & $136(49.1)$ & \multirow[t]{2}{*}{0.999} \\
\hline Female & $154(51.0)$ & $13(52.0)$ & $141(50.9)$ & \\
\hline \multicolumn{5}{|l|}{ Hospital stay setting } \\
\hline Ward & $259(85.8)$ & $1(4.0)$ & $258(93.1)$ & \multirow[t]{2}{*}{$<0.001$} \\
\hline Intensive care unit & $43(14.2)$ & $24(96.0)$ & $19(6.9)$ & \\
\hline Comorbid conditions & 199 (65.9) & $24(96.0)$ & $175(63.2)$ & 0.002 \\
\hline Diabetes mellitus & $80(26.5)$ & $13(52.0)$ & $67(24.2)$ & 0.005 \\
\hline Hypertension & $115(38.1)$ & $17(68.0)$ & $98(35.4)$ & 0.003 \\
\hline Chronic obstructive lung disease & $40(13.2)$ & $4(16.0)$ & $36(13.0)$ & 0.756 \\
\hline Coronary artery disease & $34(11.3)$ & $7(28.0)$ & $27(9.8)$ & 0.014 \\
\hline Congestive heart failure & $11(3.6)$ & $3(12.0)$ & $8(2.9)$ & 0.053 \\
\hline Chronic kidney disease & $16(5.3)$ & $3(12.0)$ & $13(4.7)$ & 0.136 \\
\hline Malignancy & $28(9.3)$ & $4(16.0)$ & $24(8.7)$ & 0.269 \\
\hline \multicolumn{5}{|l|}{ COVID-19 treatments } \\
\hline Hydroxychloroquine & $300(99.3)$ & $25(100.0)$ & $275(99.3)$ & 0.999 \\
\hline Azithromycin & $289(95.7)$ & $24(96.0)$ & $265(95.7)$ & 0.999 \\
\hline Favipiravir & $70(23.2)$ & $17(68.0)$ & $53(19.1)$ & $<0.001$ \\
\hline Tocilizumab & $9(3.0)$ & $5(20.0)$ & $4(1.4)$ & $<0.001$ \\
\hline Oseltamivir & $236(78.1)$ & $22(88.0)$ & $214(77.3)$ & 0.321 \\
\hline Low molecular weight heparin & $146(48.3)$ & $18(72.0)$ & $128(46.2)$ & 0.024 \\
\hline Broad spectrum antibiotics & $48(15.9)$ & $15(60.0)$ & $33(11.9)$ & $<0.001$ \\
\hline \multicolumn{5}{|l|}{ Laboratory parameters } \\
\hline White blood cell count $\left(\times 10^{9} / \mathrm{L}\right)$ & $6.80[0.93-34.34]$ & 11.05 [7.82-14.56] & $6.1[4.74-8.67]$ & $<0.001$ \\
\hline Neutrophil count $\left(\times 10^{9} / \mathrm{L}\right)$ & $4.50[0.59-31.63]$ & 9.4 [2.3-31.63] & $4.12[0.59-19.86]$ & $<0.001$ \\
\hline Lymphocyte count $\left(\times 10^{9} / \mathrm{L}\right)$ & $1.37[0.13-4.22]$ & $0.78[0.25-3.64]$ & $1.42[0.13-4.22]$ & $<0.001$ \\
\hline NLR & $3.1[0.6-50.0]$ & $11.6[2.4-49.2]$ & $2.9[0.6-50.0]$ & $<0.001$ \\
\hline Hemoglobin (g/dL) & $12.5[6.0-16.6]$ & $11.2 \pm 2.2$ & $12.4 \pm 1.9$ & 0.012 \\
\hline RDW $(\%)$ & 13.6 [11.7-29.4] & $15.2 \pm 1.9$ & $14.1 \pm 2.0$ & 0.015 \\
\hline Platelet count $\left(\times 10^{9} / \mathrm{L}\right)$ & 218 [34-553] & $246 \pm 83$ & $230 \pm 83$ & 0.336 \\
\hline MPV (fL) & $10.0[1.0-16.1]$ & $10.3[1.0-14.5]$ & $10.0[1.0-16.1]$ & 0.019 \\
\hline C-reactive protein (mg/dL) & $2.7[0.1-34.0]$ & $10.7[0.2-34.0]$ & $2.2[0.1-34.0]$ & $<0.001$ \\
\hline Procalcitonin (ng/mL) & $0.1[0.0-54.6]$ & $0.7[0.1-13.4]$ & $0.1[0.0-54.6]$ & $<0.001$ \\
\hline D-dimer (ng/mL) & 793 [20-19400] & $3660[210-9650]$ & 713 [20-19400] & $<0.001$ \\
\hline LDH (U/L) & 294.5 [90.0-1917.0] & $289.0[230.0-376.0]$ & $463.0[368.0-626.0]$ & $<0.001$ \\
\hline Ferritin $(\mathrm{ng} / \mathrm{mL})$ & $279.0[2.1-4835.0]$ & $813[87-3295]$ & $273[2-4835]$ & $<0.001$ \\
\hline Fibrinogen (mg/dL) & $422[30-2716]$ & $409.0[317.0-525.0]$ & $627.0[492.0-706.0]$ & $<0.001$ \\
\hline Prothrombin time (seconds) & 14.8 [12.4-32.3] & $17.1 \pm 3.9$ & $15.1 \pm 2.0$ & $\mathbf{0 . 0 1 8}$ \\
\hline INR & $1.1[0.9-38.5]$ & $1.2[1.1-1.4]$ & $1.1[1.0-1.2]$ & $<0.001$ \\
\hline aPTT (seconds) & $31.1[21.3-50.2]$ & $35.0 \pm 6.0$ & $31.2 \pm 3.7$ & 0.004 \\
\hline
\end{tabular}

Boldvalues denote statistical significance at the $p \leq 0.05$ level

aPTT: activated thromboplastin time, INR: international normalized ratio, NLR: neutrophil-to-lymphocyte ratio, MPV: mean platelet volume, RDW: red cell distribution width

the survivors and non-survivors of COVID-19 patients. Elderly patients with hypertension were the most vulnerable group for mortality. Lymphopenia, leukocytosis, higher procalcitonin and NLR, and prolonged coagulation tests were associated with the development of mortality in COVID-19. 
Table 2 Number and percentage of patients with blood counts above and below the normal reference ranges (Other hematological/ inflammatory parameters presented as number and percentage above the normal reference range)

\begin{tabular}{|c|c|c|c|c|}
\hline & \multirow{2}{*}{$\begin{array}{l}\text { All patients } \\
(\mathrm{n}=302)\end{array}$} & \multicolumn{2}{|c|}{ Survival status } & \multirow[t]{2}{*}{$p$-value } \\
\hline & & $\begin{array}{l}\text { Survivor } \\
(\mathrm{n}=277)\end{array}$ & $\begin{array}{l}\text { Deceased } \\
(\mathrm{n}=25)\end{array}$ & \\
\hline \multicolumn{5}{|c|}{ White blood cell count $\left(\times 10^{9} / \mathrm{L}\right)(\%)$} \\
\hline$<4.0$ & 39 (12.9) & $38(13.7)$ & $1(4.0)$ & \multirow[t]{3}{*}{$<0.001$} \\
\hline $4.0-10.0$ & $203(67.2)$ & $193(69.7)$ & $10(40.0)$ & \\
\hline$>10.0$ & $60(19.9)$ & $46(16.6)$ & $14(56.0)$ & \\
\hline \multicolumn{5}{|c|}{ Neutrophil count $\left(\times 10^{9} / \mathrm{L}\right)(\%)$} \\
\hline$<2.0$ & $25(8.3)$ & $25(9.0)$ & $0(0.0)$ & \multirow[t]{3}{*}{$<0.001$} \\
\hline $2.0-7.0$ & $206(68.2)$ & $199(71.8)$ & $7(28.0)$ & \\
\hline$>7.0$ & $71(23.5)$ & $53(19.1)$ & $18(72.0)$ & \\
\hline \multicolumn{5}{|c|}{ Lymphocyte count $\left(\times 10^{9} / \mathrm{L}\right)(\%)$} \\
\hline$<0.8$ & $58(19.2)$ & $45(16.2)$ & $13(52.0)$ & \multirow[t]{3}{*}{$<0.001$} \\
\hline $0.8-4.0$ & $242(80.1)$ & $230(83.0)$ & $12(48.0)$ & \\
\hline$>4.0$ & $2(0.7)$ & $2(0.7)$ & $0(0.0)$ & \\
\hline \multicolumn{5}{|l|}{ Hemoglobin $(\mathrm{g} / \mathrm{dL})(\%)$} \\
\hline$<12$ & $122(40.4)$ & $107(38.6)$ & $15(60.0)$ & \multirow[t]{3}{*}{0.113} \\
\hline $12-16$ & $176(58.3)$ & $166(59.9)$ & $10(40.0)$ & \\
\hline$>16$ & $4(1.3)$ & $4(1.4)$ & $0(0.0)$ & \\
\hline \multicolumn{5}{|c|}{ Platelet count $\left(\times 10^{9} / \mathrm{L}\right)(\%)$} \\
\hline$<150$ & $43(14.2)$ & $39(14.1)$ & $4(16.0)$ & \multirow[t]{3}{*}{0.849} \\
\hline $150-450$ & $254(84.1)$ & $233(84.1)$ & $21(84.0)$ & \\
\hline$>450$ & $5(1.7)$ & $5(1.8)$ & $0(0.0)$ & \\
\hline \multicolumn{5}{|c|}{ Percent of the patients with value above normal } \\
\hline RDW (\%) & $51(16.9)$ & $46(16.6)$ & $5(20.0)$ & 0.588 \\
\hline MPV (\%) & $38(12.6)$ & $29(10.5)$ & $9(36.0)$ & 0.001 \\
\hline C-reactive protein $(\%)$ & $235(77.8)$ & $211(76.2)$ & $24(96.0)$ & 0.042 \\
\hline Procalcitonin $(\%)$ & $126(41.7)$ & $101(36.5)$ & $25(100.0)$ & $<0.001$ \\
\hline D-dimer $(\%)$ & $201(66.6)$ & $177(63.9)$ & $24(96.0)$ & 0.002 \\
\hline LDH $(\%)$ & $248(82.1)$ & $223(80.5)$ & $25(100.0)$ & 0.011 \\
\hline Ferritin $(\%)$ & $158(52.3)$ & $138(49.8)$ & $20(80.0)$ & 0.007 \\
\hline Fibrinogen $(\%)$ & $168(55.6)$ & $147(53.1)$ & $21(84.0)$ & 0.006 \\
\hline Prothrombin time $(\%)$ & $88(29.1)$ & $73(26.4)$ & $15(60.0)$ & 0.001 \\
\hline $\operatorname{INR}(\%)$ & $43(14.2)$ & 33 (11.9) & $10(40.0)$ & 0.001 \\
\hline aPTT $(\%)$ & $13(4.3)$ & $7(2.5)$ & $6(24.0)$ & $<0.001$ \\
\hline
\end{tabular}

Boldvalues denote statistical significance at the $p \leq 0.05$ level

$N L R$ neutrophil-to-lymphocyte ratio, $R D W$ red cell distribution width, $M P V$ mean platelet volume, $L D H$ lactate dehydrogenase, INR international normalized ratio, aPTT activated thromboplastin time

Normal laboratory reference values: MPV: $6-12 \mathrm{fL}, \mathrm{CRP}: 0-0.5 \mathrm{mg} / \mathrm{dL}$, procalcitonin: $<0.05 \mathrm{ng} / \mathrm{mL}$, D-dimer: 0-500 ng/mL, LDH: 125-220 U/L, ferritin: 22-275 ng/mL, Fibrinogen: 200-400 mg/dL, PT: 11-16 s, INR: $0.8-1.3$, aPTT: $25-40 \mathrm{~s}$
In a systematic review, significantly higher serum D-dimer levels were found in COVID-19 patients with acute respiratory distress syndrome (ARDS) and the subgroup of those with mortality [18]. The authors concluded a significant correlation between elevated D-Dimer levels and complications and mortality due to COVID-19. However, Favaloro et al. [19] expressed some concerns regarding the measurement and quality of reporting of D-dimer such as the measurement method, assay, cut-off values, or D-dimer units [i.e., D-dimer units (DDU) or fibrinogen equivalent units (FEU)]. Although these issues may be important in comparing the results of different studies, our results showed that higher serum D-dimer levels were significantly associated with mortality due to COVID-19. So, we think that D-Dimer monitoring in COVID-19 patients may have some critical therapeutic impacts to tailor the treatment. 
Table 3 ROC analysis of laboratory parameters for the prediction of mortality

\begin{tabular}{|c|c|c|c|c|c|c|}
\hline & AUC & Sensitivity & Specificity & Cut Off & $95 \% \mathrm{CI}$ & $p$ \\
\hline White blood cell count $(\times 109 / \mathrm{L})$ & 0.771 & 76.00 & 67.51 & $>7810$ & $0.720-0.818$ & $<0.001$ \\
\hline Neutrophil count $\left(\times 10^{9} / \mathrm{L}\right)$ & 0.823 & 76.00 & 79.78 & $>6760$ & $0.776-0.865$ & $<0.001$ \\
\hline Lymphocyte count $\left(\times 10^{9} / \mathrm{L}\right)$ & 0.723 & 72.00 & 69.31 & $\leq 1070$ & $0.669-0.773$ & $<0.001$ \\
\hline NLR & 0.861 & 72.00 & 88.45 & $>8.59$ & $0.817-0.898$ & $<0.001$ \\
\hline Hemoglobin (g/dL) & 0.656 & 48.00 & 82.67 & $\leq 10.5$ & $0.600-0.710$ & 0.013 \\
\hline RDW $(\%)$ & 0.681 & 60.00 & 67.51 & $>14.3$ & $0.626-0.734$ & 0.001 \\
\hline Platelet count $\left(\times 10^{9} / \mathrm{L}\right)$ & 0.577 & 68.00 & 49.46 & $>213,000$ & $0.519-0.633$ & 0.223 \\
\hline MPV (fL) & 0.642 & 36.00 & 93.5 & $>12.2$ & $0.585-0.696$ & 0.025 \\
\hline C-reactive protein $(\mathrm{mg} / \mathrm{dL})$ & 0.800 & 96.00 & 57.04 & $>2.9$ & $0.750-0.843$ & $<0.001$ \\
\hline Procalcitonin (ng/mL) & 0.894 & 96.00 & 77.26 & $>0.11$ & $0.854-0.926$ & $<0.001$ \\
\hline D-dimer (ng/mL) & 0.843 & 76.00 & 85.56 & $>1893$ & $0.797-0.882$ & $<0.001$ \\
\hline LDH (U/L) & 0.798 & 84.00 & 67.51 & $>341$ & $0.748-0.842$ & $<0.001$ \\
\hline Ferritin $(\mathrm{ng} / \mathrm{mL})$ & 0.803 & 72.00 & 89.53 & $>625$ & $0.753-0.846$ & $<0.001$ \\
\hline Fibrinogen (mg/dL) & 0.748 & 72.00 & 76.17 & $>531$ & $0.695-0.796$ & $<0.001$ \\
\hline Prothrombin time (seconds) & 0.703 & 68.00 & 72.92 & $>15.7$ & $0.648-0.754$ & 0.001 \\
\hline INR & 0.728 & 64.00 & 79.06 & $>1.21$ & $0.674-0.777$ & $<0.001$ \\
\hline aPTT (seconds) & 0.707 & 56.00 & 79.42 & $>33.5$ & $0.653-0.758$ & 0.001 \\
\hline
\end{tabular}

Boldvalues denote statistical significance at the $p \leq 0.05$ level

$N L R$ neutrophil-to-lymphocyte ratio, $R D W$ red cell distribution width, $M P V$ mean platelet volume, $L D H$ lactate dehydrogenase, $I N R$ international normalized ratio, $a P T T$ activated thromboplastin time

Although it is not yet entirely clear how SARS-CoV-2 activates the coagulation cascade, it is plausible to think that it might be a byproduct of cytokine storm [20]. The aPTT and PT are usually prolonged during severe COVID19 , but the amount of elevation is milder than that observed in bacterial sepsis and DIC [21]. A meta-analysis conducted by Henry et al. [22] found that patients with severe and fatal COVID-19 had significantly more elevated coagulation parameters (especially PT) than patients with non-severe disease. It has been suggested that the coagulopathy observed in patients with severe COVID-19 is a mixture of localized pulmonary thrombotic microangiopathy and low-grade DIC [9]. We detected significant prolongations in the coagulation tests in the current study, consistent with the previously published studies [21, 22]. Although the mechanisms of these changes are not fully elucidated, the significant changes in the coagulation tests, increased PT, INR and aPTT, might be regarded as useful biomarkers to guide the disease severity and virus-induced biological catastrophic cascades.

Changes in peripheral blood cell counts have been wellstudied in COVID-19. In infected patients, counts of white blood cells and neutrophils increase, whereas counts of lymphocyte and platelet decrease [22]. In a subset of patients, thrombocytopenia has been related to coagulation abnormalities (prolonged INR and aPTT) and DIC [23]. It has been postulated that several possible mechanisms might be responsible for this, including the viral cytopathic effect on lymphocytes, the suppressive effect of cytokine storm, lactic acidosis, and lymphoid organ atrophy [24-26]. Moreover, lymphopenia was associated with the development of ARDS [27, 28]. Peripheral blood neutrophilia has been shown to predict mortality in COVID-19 independently [28]. In our study, WBC and neutrophil counts were significantly higher, and lymphocyte counts were significantly lower in the deceased than those in the surviving patients. The NLR also emerged as one of the independent predictors of mortality in our COVID-19 patient cohort. This result was in line with the previously published studies [29-31]. Since NLR is calculated by considering both neutrophils and lymphocytes, the changes in the NLR values may help physicians assess the severity and prognosis of COVID-19 infections. A meta-analysis including nine studies with a total of 1,779 COVID-19 patients demonstrated that thrombocytopenia was associated with a more than five-fold increased risk of severe COVID-19. Low platelet counts were also significantly associated with increased mortality [32].

On the other hand, platelet counts were comparable between the groups in the current study. Different pathophysiological mechanisms may lead to such insignificant findings.

It seems that a reciprocal interaction exists between SARS-CoV-2 induced cytokine storm (inflammation) and 

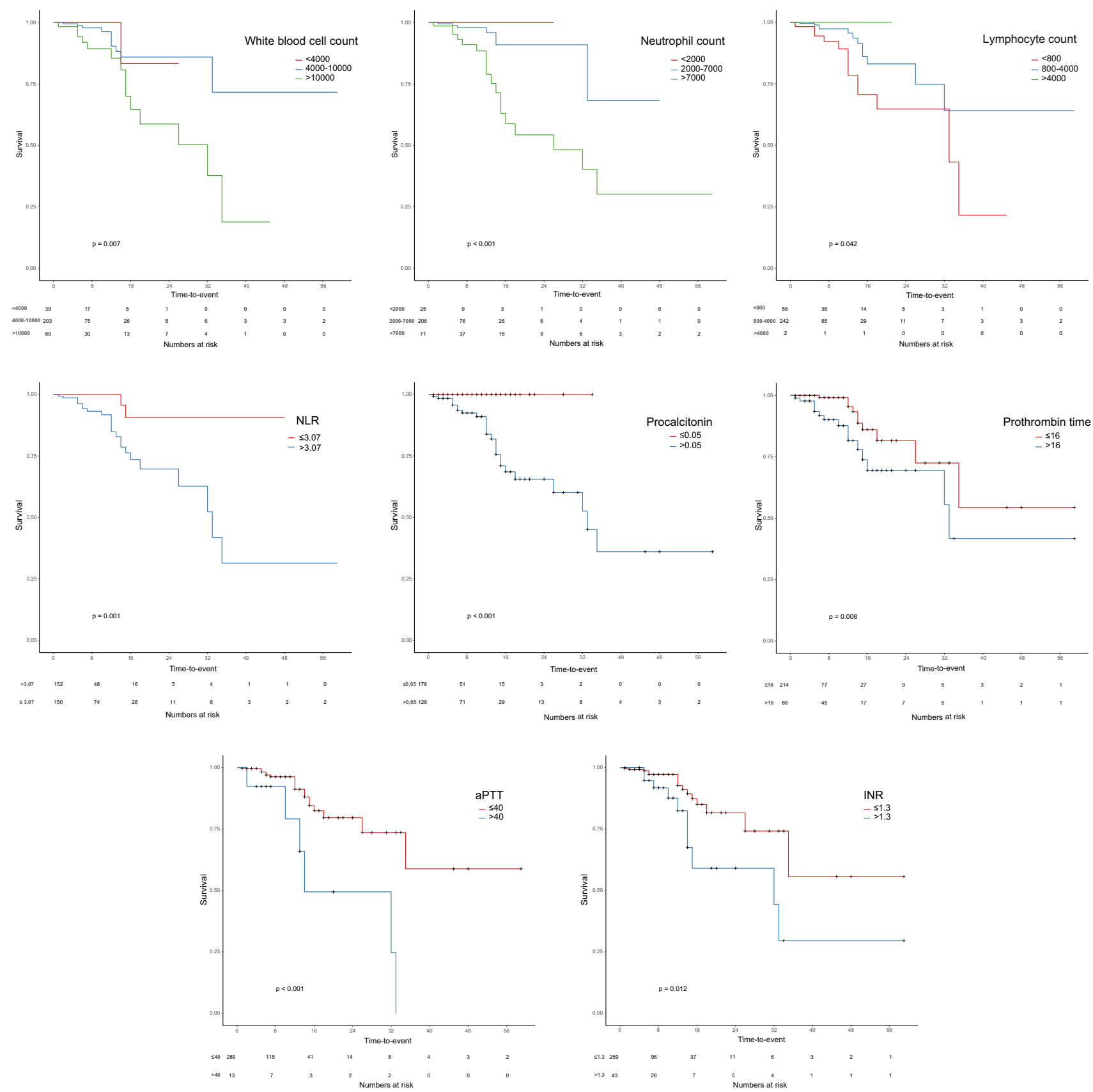

Fig. 1 Kaplan-Meier survival curves for patients with normal and abnormal values for white blood cell count, neutrophil count, lymphocyte count, neutrophil-to-lymphocyte ratio, procalcitonin, prothrombin time, aPTT, and INR

activated coagulation system [33]. In severe COVID-19, a remarkable inflammatory state is usually observed, as evidenced by increased serum CRP, interleukin-6, procalcitonin, and ferritin levels [34, 35]. Although this was a viral infection, higher procalcitonin levels increased the risk of severe COVID-19 five-fold [36]. Similarly, higher CRP levels in COVID-19 patients were associated with ARDS development, myocardial injury, and death $[28,37,38]$. A meta-analysis reported that elevated serum levels of ferritin, procalcitonin, and D-dimer were associated with more deaths, ARDS development, and need for ICU care in COVID-19 patients [39]. Bacterial infections secondary viral infections would be additional reasons to explain the elevations in these inflammatory biomarkers. Our results demonstrated that serum CRP, procalcitonin, and ferritin levels were significantly higher in the deceased COVID-19 patients, reflecting a poor composite outcome. There are controversial issues about the cut-off values of these biomarkers [39]. Thus, we recommend using CRP, ferritin, and procalcitonin changes to 
Table 4 Independent determinants of mortality according to univariate and multivariate binary logistic regression analyses

\begin{tabular}{lllll}
\hline & Crude & Crude & Adjusted & Adjusted \\
& HR $(95 \% \mathrm{CI})$ & $p$-value & HR $(95 \% \mathrm{CI})$ & $p$-value \\
\hline Age $>$ 65 years: present or absent & $3.11[1.23-7.91]$ & $\mathbf{0 . 0 1 7}$ & $3.19[1.13-9.06]$ & $\mathbf{0 . 0 2 9}$ \\
Hypertension: present vs absent & $2.66[1.14-6.18]$ & $\mathbf{0 . 0 2 3}$ & $2.99[1.03-8.7]$ & $\mathbf{0 . 0 4 4}$ \\
Chronic kidney disease: present vs absent & $3.24[0.95-11.05]$ & 0.061 & - & - \\
Diabetes mellitus: present vs absent & $2.13[0.96-4.72]$ & 0.062 & - & - \\
Congestive heart failure: present vs absent & $2.12[0.62-7.23]$ & 0.229 & - & - \\
Platelet count: high or normal & $1.00[1.00-1.00]$ & 0.175 & - & - \\
MPV: high or normal & $1.01[0.85-1.21]$ & 0.870 & & \\
NLR: high or normal & $1.05[1.03-1.08]$ & $<\mathbf{0 . 0 0 1}$ & $1.05[1.02-1.09]$ & $\mathbf{0 . 0 0 1}$ \\
C-reactive protein: high or normal & $1.05[1.01-1.09]$ & $\mathbf{0 . 0 2 2}$ & $1.03[0.97-1.09]$ & 0.350 \\
Procalcitonin: high or normal & $1.05[1.01-1.10]$ & $\mathbf{0 . 0 2 4}$ & $1.05[0.96-1.15]$ & 0.271 \\
Ferritin: high or normal & $1.00[1.00-1.00]$ & $\mathbf{0 . 0 3 4}$ & $1.00[1.00-1.00]$ & 0.831 \\
Prothrombin time: high or normal & $1.13[1.03-1.24]$ & $\mathbf{0 . 0 1 1}$ & $0.96[0.83-1.11]$ & 0.566 \\
aPTT: high or normal & $1.10[1.03-1.18]$ & $\mathbf{0 . 0 0 3}$ & $1.15[1.03-1.29]$ & $\mathbf{0 . 0 1 3}$ \\
\hline
\end{tabular}

Boldvalues denote statistical significance at the $p \leq 0.05$ level

$H R$ hazard ratio, $C I$ confidence interval, $N L R$ neutrophil-to-lymphocyte ratio, $R D W$ red cell distribution width, $M P V$ mean platelet volume, $a P T T$ activated thromboplastin time monitor the progression and improvements of COVID-19 patients.

The limitations of the current study are as follows. First, we did not assess the development of ARDS complications and acute kidney injury in the study group. Thus, we cannot confidently say that impaired inflammatory and coagulation factors are more common in complicated COVID-19. The only outcome measure of our study was in-hospital mortality. Although we evaluated the platelet count, D-dimer, and fibrinogen levels, we did not calculate the International Society on Thrombosis and Hemostasis (ISTH) score of study patients. Thus, we cannot distinguish patients who had mild-to-moderate coagulation abnormalities from those with full-scale DIC.

In conclusion, the current study showed that inflammatory, hematological, and coagulation parameters were elevated in most COVID-19 patients. While neutrophil and WBC counts increased, lymphocyte counts decreased significantly as the disease severity increased. Age $\geq 65$ years, NLR, aPTT, and hypertension were the independent predictors of mortality.

Acknowledgements We thank Gökhan Karakoç and the editors of Model Statistics Center for help with the statistical treatment of the data.

Funding This research did not receive any specific grant from funding agencies in the public, commercial, or not-for-profit sectors.

Code Availability N/A.

Data Availability N/A.

\section{Compliance with Ethical Standards}

Conflict of interest The author declares that he has no conflict of interest.

Ethics Approval The study was approved by Ümraniye Education and Research Hospital Ethics Committee (28.04.2020, \#54132726000-9041) and performed ethically in accordance with the Declaration of Helsinki.

Consent to Participate All participants were informed about the study and their consent was obtained.

Consent for Publications N/A.

\section{References}

1. Center for Systems Science and Engineering (CSSE) at Johns Hopkins University (JHU). COVID-19 Dashboard. https:// gisanddata.maps.arcgis.com/apps/opsdashboard/index.html\#/ bda7594740fd40299423467b48e9ecf6. Accessed 23 Jul 2020

2. Jain V, Yuan JM (2020) Predictive symptoms and comorbidities for severe COVID-19 and intensive care unit admission: a systematic review and meta-analysis. Int J Public Heal 65:533-546

3. Guan WJ, Liang WH, Zhao Y, Liang HR, Chen ZS, Li YM et al (2020) Comorbidity and its impact on 1590 patients with COVID-19 in China: a nationwide analysis. Eur Respir J 55(5):2000547. https://doi.org/10.1183/13993003.00547-2020

4. Guan WJ, Ni ZY, Hu Y, Liang WH, Ou CQ, He JX et al (2020) Clinical characteristics of coronavirus disease 2019 in China. N Engl J Med 382:1708-1720

5. Shang W, Dong J, Ren Y, Tian M, Li W, Hu J et al (2020) The value of clinical parameters in predicting the severity of COVID19. J Med Virol. https://doi.org/10.1002/jmv.26031

6. Shang Y, Liu T, Wei Y, Li J, Shao L, Liu M et al (2020) Scoring systems for predicting mortality for severe patients with COVID- 
19. EClinicalMedicine 24:100426. https://doi.org/10.1016/j. eclinm.2020.100426

7. Hu H, Yao N, Qiu Y (2020) Comparing rapid scoring systems in mortality prediction of critically 11 patients with novel coronavirus disease. Acad Emerg Med 27:461-468

8. Tang N, Li D, Wang X, Sun Z (2020) Abnormal coagulation parameters are associated with poor prognosis in patients with novel coronavirus pneumonia. J Thromb Haemost 18:844-847

9. Levi M, Thachil J, Iba T, Levy JH (2020) Coagulation abnormalities and thrombosis in patients with COVID-19. Lancet Haematol 7:e438-e440

10. Fei Y, Tang N, Liu H, Cao W (2020) Coagulation dysfunction: a hallmark in COVID-19. Arch Pathol Lab Med. https://doi.org/10. 5858/arpa.2020-0324-SA

11. Guevara-Noriega KA, Lucar-Lopez GA, Nuñez G, RiveraAguasvivas L, Chauhan I (2020) Coagulation panel in patients with SARS-CoV2 infection (COVID-19). Ann Clin Lab Sci 50:295-298

12. Wang J, Saguner AM, An J, Ning Y, Yan Y, Li G (2020) Dysfunctional Coagulation in COVID-19: from cell to bedside. Adv Ther 37:3033-3039

13. Rico-Mesa JS, Rosas D, Ahmadian-Tehrani A, White A, Anderson AS, Chilton R (2020) The role of anticoagulation in COVID-19-induced hypercoagulability. Curr Cardiol Rep 22:53. https://doi.org/10.1007/s11886-020-01328-8

14. Boccia M, Aronne L, Celia B, Mazzeo G, Ceparano M, D’Agnano $\mathrm{V}$ et al (2020) COVID-19 and coagulative axis: review of emerging aspects in a novel disease. Monaldi Arch Chest Dis 90(2):271-276. https://doi.org/10.4081/monaldi.2020.1300

15. Pan F, Zheng C, Ye T, Li L, Liu D, Li L et al (2020) Different computed tomography patterns of Coronavirus Disease 2019 (COVID-19) between survivors and non-survivors. Sci Rep 10:11336. https://doi.org/10.1038/s41598-020-68057-4

16. Li B, Li X, Wang Y, Han Y, Wang Y, Wang C et al (2020) Diagnostic value and key features of computed tomography in Coronavirus Disease 2019. Emerg Microbes Infect 9:787-793

17. Hani C, Trieu NH, Saab I, Dangeard S, Bennani S, Chassagnon G et al (2020) COVID-19 pneumonia: a review of typical CT findings and differential diagnosis. Diagn Interv Imaging 101:263-268

18. Vidali S, Morosetti D, Cossu E, Luisi ML, Pancani S, Semeraro $\mathrm{V}$ et al (2020) D-dimer as an indicator of prognosis in SARSCoV-2 infection: a systematic review. ERJ Open Res 6(2):00260-02020. https://doi.org/10.1183/23120541.002602020

19. Favaloro EJ, Thachil J (2020) Reporting of D-dimer data in COVID-19: some confusion and potential for misinformation. Clin Chem Lab Med 58:1191-1199

20. Marietta M, Coluccio V, Luppi M (2020) COVID-19, coagulopathy and venous thromboembolism: more questions than answers. Intern Emerg Med, 1-13. do1:https://doi.org/10.1007/ s11739-020-02432-x

21. Iba T, Levy JH, Levi M, Thachil J (2020) Coagulopathy in COVID-19. J Thromb Haemost. https://doi.org/10.1111/jth.14975

22. Henry BM, de Oliveira MHS, Benoit S, Plebani M, Lippi G (2020) Hematologic, biochemical and immune biomarker abnormalities associated with severe illness and mortality in coronavirus disease 2019 (COVID-19): a meta-analysis. Clin Chem Lab Med 58:1021-1028

23. Bao C, Tao X, Cui W, Yi B, Pan T, Young KH et al (2020) SARS-CoV-2 induced thrombocytopenia as an important biomarker significantly correlated with abnormal coagulation function, increased intravascular blood clot risk and mortality in
COVID-19 patients. Exp Hematol Oncol 9:16. https://doi.org/10. 1186/s40164-020-00172-4

24. Terpos E, Ntanasis-Stathopoulos I, Elalamy I, Kastritis E, Sergentanis TN, Politou M et al (2020) Hematological findings and complications of COVID-19. Am J Hematol 95:834-847

25. Aggarwal S, Gollapudi S, Gupta S (1999) Increased TNF-alphainduced apoptosis in lymphocytes from aged humans: changes in TNF-alpha receptor expression and activation of caspases. J Immunol 162:2154-2161

26. Chan JF, Zhang AJ, Yuan S, Poon VK, Chan CC, Lee AC, et al (2020) Simulation of the clinical and pathological manifestations of Coronavirus Disease 2019 (COVID-19) in golden Syrian hamster model: implications for disease pathogenesis and transmissibility. Clin Infect Dis. 2020. do1: https://doi.org/10.1093/cid/ ciaa325

27. Huang C, Wang Y, Li X, Ren L, Zhao J, Hu Y, Zhang L et al (2020) Clinical features of patients infected with 2019 novel coronavirus in Wuhan. China Lancet 395:497-506

28. Wu C, Chen X, Cai Y, Zhou X, Xu S, Huang H et al (2020) Risk factors associated with acute respiratory distress syndrome and death in patients with coronavirus disease 2019 pneumonia in Wuhan, China. JAMA Intern Med 180:934-943

29. Liu Y, Du X, Chen J, Jin Y, Peng L, Wang HH et al (2020) Neutrophil-to-lymphocyte ratio as an independent risk factor for mortality in hospitalized patients with COVID-19. J Infect 81:e612. https://doi.org/10.1016/j.jinf.2020.04.002

30. Kerboua KE (2020) NLR: A cost-effective nomogram to guide therapeutic interventions in COVID-19. Immunol Invest 1-9. do1:https://doi.org/10.1080/08820139.2020.1773850

31. Tatum D, Taghavi S, Houghton A, Stover J, Toraih E, Duchesne J (2020) Neutrophil-to-lymphocyte ratio and outcomes in Louisiana Covid-19 patients. Shock. https://doi.org/10.1097/SHK. 0000000000001585

32. Lippi G, Plebani M, Henry BM (2020) Thrombocytopenia is associated with severe coronavirus disease 2019 (COVID-19) infections: a meta-analysis. Clin Chim Acta 506:145-148

33. Jose RJ, Manuel A (2020) COVID-19 cytokine storm: the interplay between inflammation and coagulation. Lancet Respir Med 8:e46-e47. https://doi.org/10.1016/S2213-2600(20)30216-2

34. Chen G, Wu D, Guo W, Cao Y, Huang D, Wang H et al (2020) Clinical and immunological features of severe and moderate coronavirus disease 2019. J Clin Invest 130:2620-2629

35. Colafrancesco S, Alessandri C, Conti F, Priori R (2020) COVID19 gone bad: a new character in the spectrum of the hyperferritinemic syndrome? Autoimmun Rev 19:102573

36. Lippi G, Plebani M (2020) Procalcitonin in patients with severe coronavirus disease 2019 (COVID-19): a meta-analysis. Clin Chim Acta 505:190-191

37. Deng Y, Liu W, Liu K, Fang YY, Shang J, Zhou L et al (2020) Clinical characteristics of fatal and recovered cases of coronavirus disease 2019 in Wuhan, China: a retrospective study. Chin Med J 133:1261-1267

38. Shi S, Qin M, Shen B, Cai Y, Liu T, Yang F et al (2020) Association of cardiac injury with mortality in hospitalized patients with COVID-19 in Wuhan, China. JAMA Cardiol 5:802-810

39. Huang I, Pranata R, Lim MA, Oehadian A, Alisjahbana B (2020) C-reactive protein, procalcitonin, D-dimer, and ferritin in severe coronavirus disease-2019: a meta-analysis. Ther Adv Respir Dis 14. do1:https://doi.org/10.1177/1753466620937175

Publisher's Note Springer Nature remains neutral with regard to jurisdictional claims in published maps and institutional affiliations. 